\title{
Dynamic Response of Electromagnetic Launcher's Rail Subjected to Cosine Pressure
}

\author{
Wen Liu \\ School of Sciences \\ Yanshan University \\ Qinhuangdao 066004, China \\ E-mail: liuwen1961@hotmail.com \\ Ligong Zhang (Corresponding author) \\ School of Sciences \\ Yanshan University \\ Qinhuangdao 066004, China \\ E-mail: zhanglg19850104@163.com
}

Xiangzhong Bai

School of Civil Engineering \& Mechanics

Yanshan University

Qinhuangdao 066004, China

Feng Liu

School of Sciences

Yanshan University

Qinhuangdao 066004, China

Received: June 30, 2011

Accepted: August 3, 2011

doi:10.5539/cis.v4n5p59

This project is supported by National Natural Science Foundation of China (Grant NO.50875230).

The Excellent Going Abroad Experts' Training Program in Hebei Province.

\begin{abstract}
In order to extend the rail life and improve the firing accuracy, the electromagnetic launcher's rail can be modeled as a beam on elastic foundation with simply supported beam by moving load. Euler beam theory is applied to build the Mechanical model and the analytical solution of the equation subjected to cosine magnetic pressure is derived in detail, which has successfully avoided the errors which are caused by using the uniform pressure to approximately replace the variable force. Numerical analysis of the dynamic response on rail by the MATLAB software shows that as the exciting frequency increases, the peek of the maximal deflection and vibration velocity increase gradually. Taking the same speed of load into account, the dynamic response of rail is obviously smaller than constant force. Therefore the reliable theory basis is provided for the design and control the rail, which promotes the practical application of the electromagnetic Launcher.
\end{abstract}

Keywords: Electromagnetic Launcher's rail, Cosine pressure, Analytical solutions, La-grange equation, Exciting frequency, Dynamic response

\section{Introduction}

Most launcher belong to chemical transmitter, such as artillery and rocket, the development of new technology produced EML (Electromagnetic Launch) (Fair H D, 2007). As a new concept weapon, the electromagnetic rail 
gun broken through the limitation of projectile velocity by the traditional chemical energy, so it has a huge application in the military field. With the development of new technology and new material, the research of launcher, launching weight, projectile velocity and high efficiency power source in the electromagnetic rail gun have reached a series of achievement in recent years. By 2002, the United States and Brigade had cooperated to launch projectile weighted $3 \sim 4 \mathrm{~kg}$ to a initial velocity of $3 \mathrm{~km} / \mathrm{s}$ with power of $32 \mathrm{MJ}$ capacitor bank (Smith A N, 2005, Fair H D, 2005 and Sympathy S S, 2004). Other countries have also made great progress in studying of the electromagnetic technology. The electromagnetic rail gun has wide application prospect in many areas, such as scientific experiment, weapon equipment, missile defence system, launching, aeronautic catapults, rocket and satellite etc.

As early as 1926, Timoshenko researched the vibration of beam at moving load, Michaltsos $G$ and Sophianopoulos D researched the effect of moving mass and other parameters on the dynamic response of a simply supported beam. T.Tzeng used the elastic foundation beam to build mechanical model of the electromagnetic rail gun and deduced the solve process of governing equation. HU Yuwei analyzed the theoretical built model and simulation analysis for the work of the process of electromagnetic rail gun. WANG Sheng adopted the Fourier transform to study displacement field due to a moving load on Euler beam resting on an elastic half-space. FANG Qing researched the dynamic response of a rigid supported beam.

However, the uniform pressure used to replace the variable force, and the damping force to the response of beam was ignored in the above researches. There is no doubt that the calculation of this kind of situation has the defects of mechanics and calculation of mechanical. As a high-tech and high-precision electromagnetic launcher, accurate theoretical analysis and calculation in engineering are require. But until now, no researcher has given any exact analytical solution, thus further analytic solution of the equation subjected to variable pressure is of great significance. In fact, it is of theoretical value to research the theoretical analytic solution of various disciplines, including the analytical solution of practical engineering problems. On the one hand, its mechanical picture can be completely stated; on the other hand it can be used as a standard solution, to widely produce variety of numerical solution. The dynamic response of a simply supported beam at a moving load has a lot of background in practical engineering, but dynamic response of electromagnetic Launcher's rail subjected to cosine pressure has not been analyzed. The electromagnetic Launcher's rail produces vibration which can not be ignored at a moving load. If the moving load is at the most unfavorable position of static and satisfies condition of resonance, a larger dynamic response of electromagnetic Launcher's rail will occur, and the electromagnetic Launcher's rail will be destroyed. Hence it is necessary to analyse the dynamic response of electromagnetic Launcher's rail.

In this paper, considering the cosine pressure and damping force, regarding the rail as simply supported beam on the elastic foundation( LIU Wen and SHAN Rui, 2009), a mechanic model which is under the effect of moving load is proposed. Moreover, making use of variable method and the Lagrange equation which considering the damping force, the analytical solution of the governing equation is derived, the displacement response and velocity response are researched, and the influence of the dynamic response of electromagnetic Launcher's rail is analyzed at exciting frequency of cosine pressure.

\section{Mechanical Model}

Figure 1 shows a schematic of an electromagnetic railgun composed of power source, rail, armature and projectile. When the electric current of armature goes through the rail, it forms a strong magnetic field in the area of their encirclement. With the reaction by the magnetic field and the electric current, it emerges powerful electromagnetic force, which pushes the armature and projectile to do the accelerating motion along the rail till the projectile is launched out of the rail.

Figure 2 is the mechanical model of the railgun-simply supported beam partially subjects to nonlinear load in a time-varying region sitting on the elastic foundation. Considering the effect of the beam by the damping force and basing on the Euler beam theory, we obtain the governing equation of elastic foundation beam by moving load which is a transient fourth-order differential equation as follows (Liu Wen, Li Min and Bai Xiang zhong, 2010):

$$
m \frac{\partial^{2} w}{\partial t^{2}}+E I \frac{\partial^{4} w}{\partial x^{4}}+k w+c \frac{\partial w}{\partial t}=p(x, t)
$$

Where $w$ is the deflection, $m=\rho b h$ is the mass per unit length, $\rho$ is the density of rail material, $b$ and $h$ are respectively the width and thickness of the rail, $E I$ is the bending stiffness of beam, $k$ is the elastic constant, $c$ is the damping coefficient. The function $p(x, t)=q(t)[1-H(x-v t)]$ in (1), represents the magnetic pressure front 
traveling along the rail with velocity $v$ represented by a Heaviside step function $H(x-v t)$ (REN Xingming and QIN Weiyang, 2006), and $q(t)=q \cos (\omega t), \omega$ is the exciting frequency of cosine pressure.

\section{Solution of the Mechanical Model}

According as the vibration of electromagnetic Launcher's rail is stacked by principal vibrations, the solution of the governing equation (1) can be expressed as follows:

$$
w(x, t)=\sum_{i} \phi_{i}(t) \theta_{i}(x)
$$

Where $\theta_{i}(x)$ is function of vibration at a boundary condition, $\phi_{i}(t)$ is function of time.

Function of vibration can be obtained by the homogeneous equation of equation (1). The homogeneous equation is a fourth-order partial differential equation; we solve it by the method of variable separation.

The solution of the homogenous equation of (1) can be expressed as follows:

$$
w(x, t)=\phi(t) \theta(x)
$$

Substituting (3) into the homogeneous equation of (1):

$$
\frac{E I}{c m} \frac{\frac{\partial^{4} \theta}{\partial x^{4}}}{\theta}+\frac{k}{c m}=-\frac{\frac{\partial^{2} \phi}{\partial t^{2}}}{c \phi}-\frac{\frac{\partial \phi}{\partial t}}{m \phi}
$$

From equation (4), let:

That is

$$
\frac{E I}{c m} \frac{\frac{\partial^{4} \theta}{\partial x^{4}}}{\theta}+\frac{k}{c m}=\lambda^{2}
$$

Where

$$
\frac{\partial^{4} \theta}{\partial x^{4}}-\beta^{4} \theta=0
$$

$$
\beta^{4}=\left(\lambda^{2}-\frac{k}{c m}\right) \frac{c m}{E I}
$$

Based on the boundary condition of the simple beam, the solution of equation (6) can be expressed as follows (ZHU Shijian, 2006):

$$
\theta_{i}(x)=\sqrt{\frac{2}{m L}} \sin \frac{n \pi}{L} x \quad(n=1,2,3 \cdots)
$$

In terms of the orthogonality of $\theta_{i}(x)$ (Jerome T T, 2005), we obtain:

$$
\int_{0}^{L} \theta_{i} \theta_{j} \mathrm{~d} x= \begin{cases}0 & \text { 当 } i \neq j \\ 1 & \text { 当 } i=j\end{cases}
$$

Considering the orthogonality of vibration, the kinetic energy of the beam $T$ can be expressed as follows:

$$
T=\frac{1}{2} \sum_{i} M_{i}\left(\frac{\partial \phi_{i}}{\partial t}\right)^{2}
$$

Where $M_{i}=\int_{0}^{L} m \theta_{i}^{2}(x) \mathrm{d} x$ represents the general mass of the beam.

The total strain energy of the beam $U$ is consisted by the strain energy $U_{b}$ of the beam, and the strain energy $U_{f}$ of the foundation.

$$
U=U_{b}+U_{f}=\frac{1}{2} \frac{E I}{m} \sum_{i} \beta_{i}^{4} M_{i} \phi_{i}^{2}+\frac{k}{2 m} \sum_{i} M_{i} \phi_{i}^{2}=\frac{1}{2}\left[\sum_{i} M_{i} \phi_{i}^{2}\left(\frac{E I}{m} \beta_{i}^{4}+\frac{k}{m}\right)\right]=\frac{1}{2}\left[\sum_{i} c M_{i} \phi_{i}^{2} \lambda_{i}^{2}\right]
$$

Where

$$
U_{b}=\frac{1}{2} \int_{0}^{L} E I\left(\frac{\partial^{2} w(x, t)}{\partial x^{2}}\right)^{2} \mathrm{~d} x=\frac{1}{2} \frac{E I}{m} \sum_{i} \beta_{i}^{4} M_{i} \phi_{i}^{2}, \quad U_{f}=\frac{1}{2} \int_{0}^{L} k w^{2}(x, t) \mathrm{d} x=\frac{k}{2 m} \sum_{i} M_{i} \phi_{i}^{2}
$$

The dissipation function $G$ can be expressed as: 


$$
G=-\frac{1}{2} \int_{0}^{l} c\left(\frac{\partial w}{\partial t}\right)^{2} \mathrm{~d} x=-\frac{c}{2 m} \sum_{i} M_{i}\left(\frac{\partial \phi_{i}}{\partial t}\right)^{2}
$$

The virtual work done by the magnetic pressure $p(x, t)=q(t)[1-H(x-v t)]$ in a virtual displacement $\delta \varphi_{i}$ can be expressed as follows:

$$
\partial W=\int_{0}^{L} p(x, t) \delta w_{i} \mathrm{~d} x=\sum_{i} \partial \phi_{i} Q_{i}
$$

Where we define as the generalized force

$$
Q_{i}=\int_{0}^{L} p(x, t) \theta_{i}(x) \mathrm{d} x=\int_{0}^{v t} q \cos (\omega t) \sqrt{\frac{2}{m L}} \sin \frac{n \pi x}{L} \mathrm{~d} x=\frac{q L}{i \pi} \sqrt{\frac{2}{m L}} \cos (\omega t)\left(1-\cos \frac{n \pi v t}{L}\right)
$$

Substituting $T, U, G, Q_{i}$ into the Baggage equation including the damping force

$$
\frac{\mathrm{d}}{\mathrm{d} t}\left(\frac{\partial T}{\frac{\partial \phi_{i}}{\partial t}}\right)-\frac{\partial T}{\partial \phi_{i}}+\frac{\partial U}{\partial \phi_{i}}-\frac{\partial G}{\frac{\partial \phi_{i}}{\partial t}}=Q_{i}
$$

We obtain an ordinary differential equation:

Where:

$$
\frac{\partial^{2} \phi_{i}}{\partial t^{2}}+\frac{c}{m} \frac{\partial \phi_{i}}{\partial t}+c \lambda_{i}^{2} \phi_{i}=\frac{Q_{i}(t)}{M_{i}}=F(t)
$$

The general solution of equation (13) is

$$
\phi_{i}(t)=\frac{m}{R}\left[\frac{\partial \phi_{i}(0)}{\partial t}+\frac{c+R}{2 m} \phi_{i}(0)\right] e^{\frac{-c+R}{2 m} t}+\frac{m}{R}\left[-\frac{\partial \phi_{i}(0)}{\partial t}+\frac{-c+R}{2 m} \phi_{i}(0)\right] e^{\frac{-c-R}{2 m} t}+\frac{m}{R} \int_{0}^{t} F(\xi)\left[e^{\frac{-c+R}{2 m}(t-\xi)}-e^{\frac{-c-R}{2 m}(t-\xi)}\right] \mathrm{d} \xi
$$

Consequently, the general solution of (1) can be expressed as follows:

$$
\begin{aligned}
w(x, t) & =\sum_{i} \theta_{i}(x) \phi_{i}(t)=\sum_{i} \sqrt{\frac{2}{m L}} \sin \frac{n \pi}{L} x\left\{\frac{m}{R}\left[\frac{\partial \phi(0)}{\partial t}+\frac{c+R}{2 m} \phi(0)\right] e^{\frac{-c+R}{2 m} t}+\frac{m}{R}\left[-\frac{\partial \phi(0)}{\partial t}+\frac{-c+R}{2 m} \phi(0)\right] e^{\frac{-c-R}{2 m} t}\right. \\
& \left.+\frac{m}{R} \int_{0}^{t} \frac{q L}{M_{i} n \pi} \sqrt{\frac{2}{m L}} \cos (\omega \xi)\left(1-\cos \frac{n \pi v \xi}{L}\right)\left[e^{\frac{-c+R}{2 m}(t-\xi)}-e^{\frac{-c-R}{2 m}(t-\xi)}\right] \mathrm{d} \xi\right\}
\end{aligned}
$$

The initial conditions are as:

We obtain:

$$
\left.\phi(t)\right|_{t=0}=0, \partial \phi(t) /\left.\partial t\right|_{t=0}=0
$$

$$
\phi_{i}(t)=\frac{m}{R} \int_{0}^{t} \frac{q L}{M_{i} n \pi} \sqrt{\frac{2}{m L}} \cos (\omega \xi)\left(1-\cos \frac{n \pi v \xi}{L}\right)\left[e^{\frac{-c+R}{2 m}(t-\xi)}-e^{\frac{-c-R}{2 m}(t-\xi)}\right] \mathrm{d} \xi
$$

According, substituting the solution of (15) into the equation (14), so we can get the solution $w(x, t)$ of (1). The velocity response of electromagnetic Launcher's rail can be further derived from $w(x, t)$, which provides basis for the overall investigation of the dynamic response of electromagnetic raisin.

\section{Numerical simulation and analysis}

In view of the different materials and the unsmooth surface of electromagnetic Launcher's rail, the moving load is regarded as exciting force with a frequency, so the electromagnetic Launcher's rail produces vibration which cannot be ignored at a moving load. If the moving load is at the most unfavorable position of static and satisfies condition of resonance, a larger dynamic response of electromagnetic Launcher's rail will occur, and the precision of Launcher will be badly affected. Hence it is necessary to analyze and compared the dynamic response of electromagnetic Launcher's rail. For cosine pressure, in order to prevent ruin form a big sudden pressure, the time of effect is confirmed at the moment that the cosine pressure is gradually increasing. Let $q(t)=q \cos (\omega t+3 \pi / 2)$. The rail is divided into meshes (LOU Ping and ZENG Qingyuan, 2004), the node of meshes is 201, and the node of 101 was chosen as the node of the dynamic response.

The parameters are as follow (Anthony J J, Francis C M, 2006): 
(1) The parameters of rail: the material Young modulus $E=120 \mathrm{GPa}$, the elastic constant $k=2.532 \times 10^{6} \mathrm{~Pa}$, the density of rail material $\rho=8.7 \times 10^{5} \mathrm{~kg} / \mathrm{m}$, the damping coefficient $c=1.3 \times 10^{5} \mathrm{Ns} / \mathrm{m}$.

(2) The geometric size of rail: the length of rail $L=2 \mathrm{~m}$, the width of rail $b=3 \times 10^{-2} \mathrm{~m}$, the thickness of rail $h=1 \times 10^{-2} \mathrm{~m}$.

(3) The parameters of load: the load collection degree $q=110 \mathrm{MPa}$, the magnetic harmonic pressure $q(t)=q \sin (\omega t) \mathrm{MPa}$, exciting frequency $\omega=10 \mathrm{~Hz}, \omega=50 \mathrm{~Hz}, \omega=60 \mathrm{~Hz}, \omega=100 \mathrm{~Hz}, \omega=1000 \mathrm{~Hz}$, $\omega=2000 \mathrm{~Hz}$, the maxima speeds of load, it is the exit velocity of projectile $v=1000 \mathrm{~m} / \mathrm{s}, v=1500 \mathrm{~m} / \mathrm{s}$, $v=2000 \mathrm{~m} / \mathrm{s}$.

\subsection{The displacement response of railgun}

When the load passing over rail with the speed of $v=1000 \mathrm{~m} / \mathrm{s}$, the node 101 deflection-time curve at different exciting frequencies is shown in Figure 3 and Figure 4. Based on the Figure 3 and Figure 4, as the exciting frequency increases, the peek of the maximal deflection increases gradually under cosine pressure; when the exciting frequency is small, the maximal deflection occur at the moment that cosine pressure will leave the rail; when the exciting frequency is larger, the resonance will occur.

When the load passing over rail at same exciting frequencies, the node deflection-time curve at different speeds of load is shown in Figure 5-(a),(b). Based on the comparison of Figure 5-(a),(b), If the exciting frequency remains unchanged, the maximal deflection occur at the moment that cosine pressure will leave the rail; As the speeds of load increases, the time decreases while the load passes over rail and the maximal deflection also decreases gradually, but deflection-time curve has no obvious change; when the exciting frequency of cosine pressure increases, the peek of the maximal deflection increases gradually; When the projectile leaves rail and the load stops functioning to railgun, the vibration of rail will be a free vibration by a damping initial state.

Figure 6 shows the node deflection-time curve at different loads. The rail affected by the load collection degree $q$ and the magnetic cosine pressure $q \cos (50 t+3 \pi / 2)$, the maximal deflection of rail at harmonic pressure is significantly smaller than constant force with same speeds of load.

\subsection{The velocity response of railgun}

When the load passing over rail with the speed of $v=1000 \mathrm{~m} / \mathrm{s}$, the change of vibration velocity with time in the case of different exciting frequency of harmonic pressure is shown in Figure 7. Based on the Figure 7, as the exciting frequency increase, the peek of the maximal vibration velocity increases gradually under cosine pressure; the maximal vibration velocity occurs at the moment that the cosine pressure will leave the rail.

When the load passing over rail at same exciting frequencies, the vibration velocity-time curve at different speeds of load is shown in Figure 8-(a),(b). Based on the comparison of Figure 8-(a),(b), when the exciting frequency of cosine pressure increases, the peek of the maximal vibration velocity increases gradually; When the load stops functioning to railgun, the vibration of rail will be a free vibration. If the exciting frequency remains unchanged during the load passes over rail, the maximal vibration velocity occur at the moment that cosine pressure will leave the rail; as the speeds of load increases, the time decreases while the load passes over rail, the maximal vibration velocity decreases gradually, and the peek of vibration velocity also decreases gradually.

Figure 9 shows the vibration velocity-time curve at different loads The rail affected by the load collection degree $q$ and the magnetic cosine pressure $q \cos (50 t+3 \pi / 2)$, the vibration velocity of rail at harmonic pressure is significantly smaller than constant force with same speeds of load.

\section{Conclusion}

(1) Making use of variable method and the Lagrange equation including the damping force, the general solution of the homogeneous part and the analytical solution of the governing equation subjected to cosine pressure is derived, which enriched and developed the theory of elastic mechanics with the hope to lay the foundation for solving the difficulty problem of electromagnetic rail subjected to arbitrary distribution function pressures.

(2) Though the numerical simulation and analysis with MATLAB, the character of dynamic response of electromagnetic Launcher's rail was analyzed when cosine pressure with different exciting frequencies passed through the rail. As the exciting frequency increases, the peek of the maximal deflection and the peek of the maximal vibration velocity increase gradually under cosine pressure. The resonance occurs when the exciting frequency is close to natural frequency. In the case of moving load at same speeds, the maximal deflection and vibration velocity of rail at cosine pressure is smaller than constant force. 


\section{References}

Anthony J J \& Francis C M. (2006). Elastic Waves and Solid Armature Contact Pressure in Electromagnetic Launchers. Transactions on Magnetics, 3, 472-475.

Fair H D. (2005). Electromagnetic Launch Science and Technology in the United States Enters a New Era. IEEE Transactions on Magnetics, 41(1), 158-164. doi:10.1109/TMAG.2004.838744, http://dx.doi.org/10.1109/TMAG.2004.838744.

Fair H D. (2007). Progress in Electromagnetic Launch Science and Technology. IEEE Transactions on Magnetics, 2007, 43(1), 93-98. doi:10.1109/TMAG.2006.887596, http://dx.doi.org/10.1109/TMAG.2006.887596.

Fang qing \& Du maoling. (2006). Dynamic responses of an elastically supported beams with damping subjected to blast loads. Mechanics in Engineering, 28(2), 53-56.

HU Yuwei. (2007). Modeling and simulation of electromagnetic rail gun system: [Dissertation for the Master Degree]. Harbin Institute of Technology.

Jerome T Tzeng \& Wei S. (2007). Dynamic Response of Cantilevered Rail Guns Attributed to Projectile/Gun Interaction-Theory. IEEE Transactions on Magnetics, 43, 207-213. doi:10.1109/TMAG.2006.887444, http://dx.doi.org/10.1109/TMAG.2006.887444.

Liu Wen \& Shan Rui. (2009). Mathematic Model and Analytic Solution for a Cylinder Subject to Exponential Function. Chinese Journal of Mechanical Engineering, 22(4), 587-593. doi:10.3901/CJME.2009.04.587, http://dx.doi.org/10.3901/CJME.2009.04.587.

LIU Wen, LI Min \& BAI Xiangzhong. Deformation Calculating of Electromagnetic Launcher's Rail Subjected to Exponential Magnetic Pressure. Harbin Institute of Technology, 42(8), 1336-1340.

LOU Ping \& ZENG Qingyuan. (2004). Finite element analysis of slab track subjected to moving load. Journal of Traffic and Transportation Engineering, 3(1), 29-33.

Michaltsos G, Sophianopoulos D \& Kounadis A N. (1996). The effect of a moving mass and other parameters on the dynamic response of a simply supported beam. Journal of sound and Vibratio, 191(3), 357-362.

REN Xingming \& QIN Weiyang. (2006). Basic of Engineering Vibration. China. Machine Press, 123-138.

Satapathy S S, McNab I R \& Erengil M E. (2004). Design of an 8-MJ Integrated Launch Package. IEEE Proceedings of 2004, 12th Symposium on Electromagnetic launch Technology, 405-410, doi:10.1109/ELT.2004.1398114, http://dx.doi.org/10.1109/ELT.2004.1398114.

Smith A N, Ellis R L \& Bernardes J S. (2005). Thermal Management and Resistive Rail Heating Large-scale Naval Electro-magnetic Launcher. IEEE Transactions on Magnetizes, 41(1), 235-240. 10.1109/TMAG.2004.839269, http://dx.doi.org/10.1109/TMAG.2004.839269.

Wang sheng. (2007). Displacement field due to a moving load on Euler beam resting on an elastic half-space: [Dissertation for the Master Degree].Harbin Institute of Technology.

ZHU Shijian. (2006). The theory vibration and the techniques of vibration isolation. National Defence Industry Press, 36-66. 


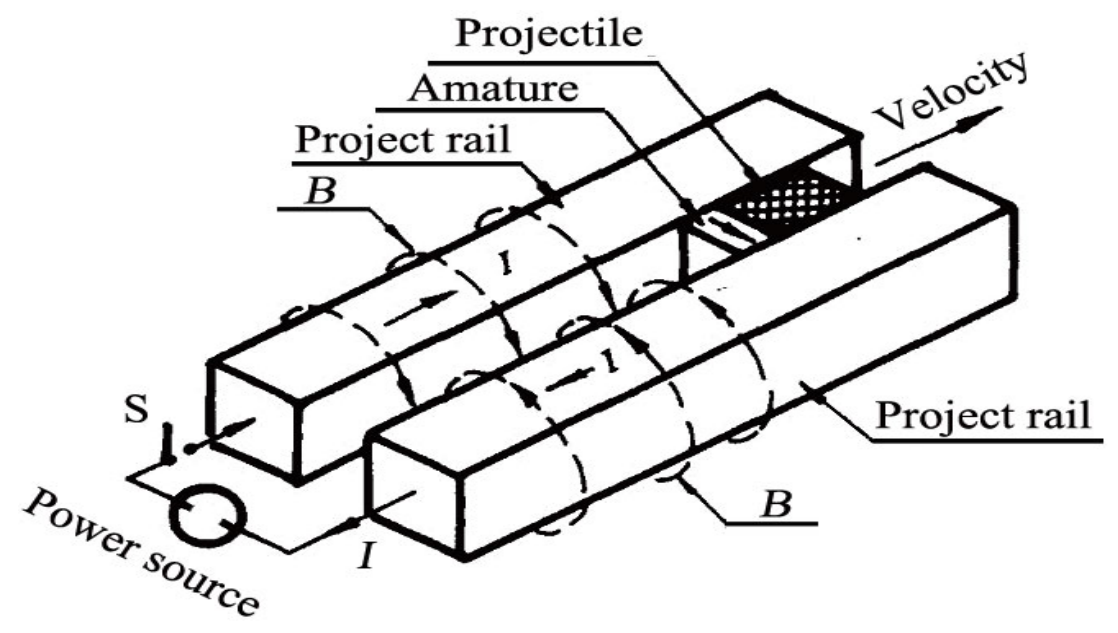

Figure 1. The general diagram of the railgun

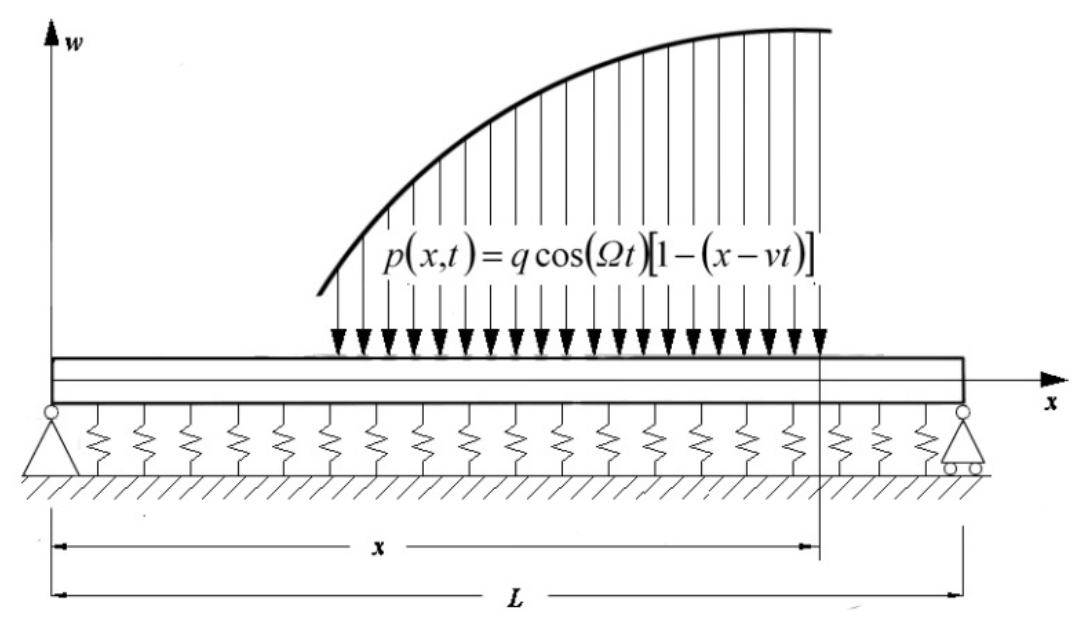

Figure 2. The rail is modeled as a beam on elastic foundation 


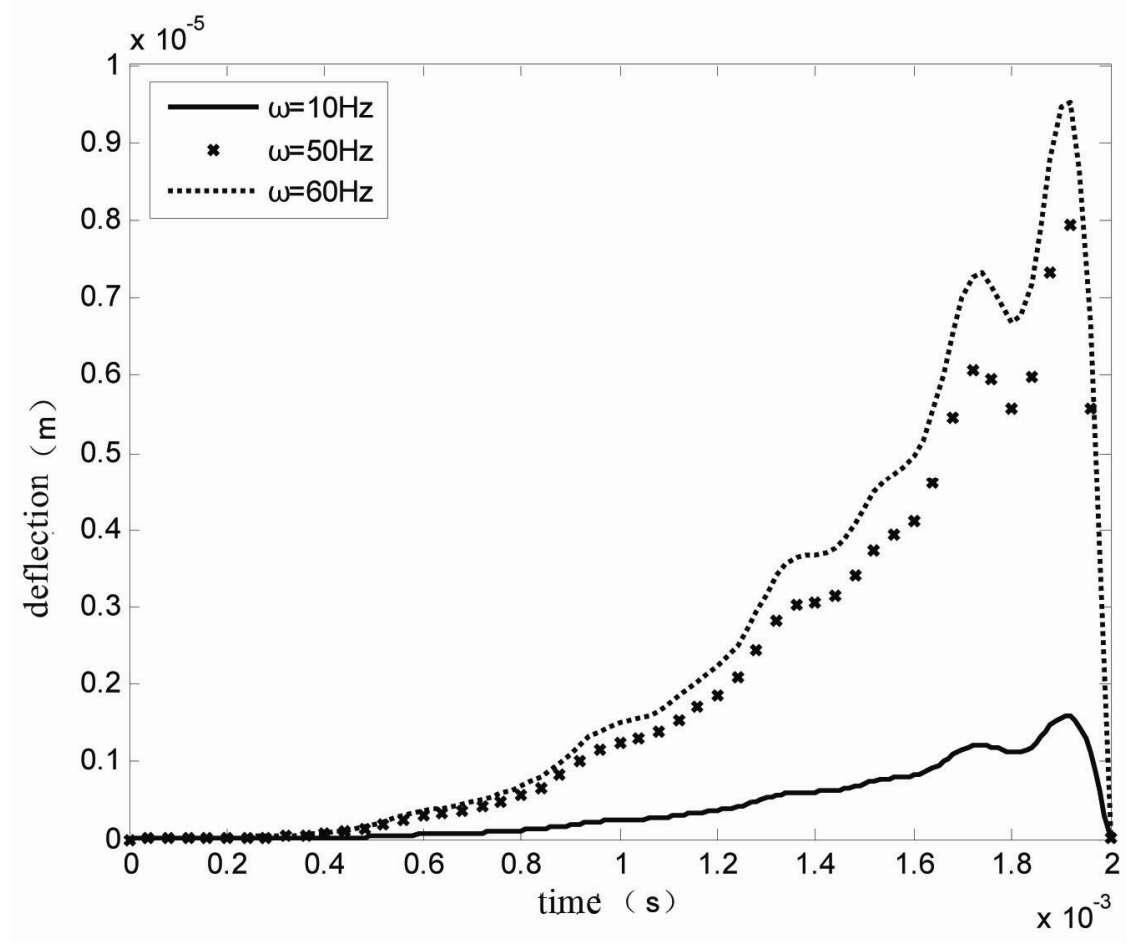

Figure 3. Node deflection-time curve at different frequencies

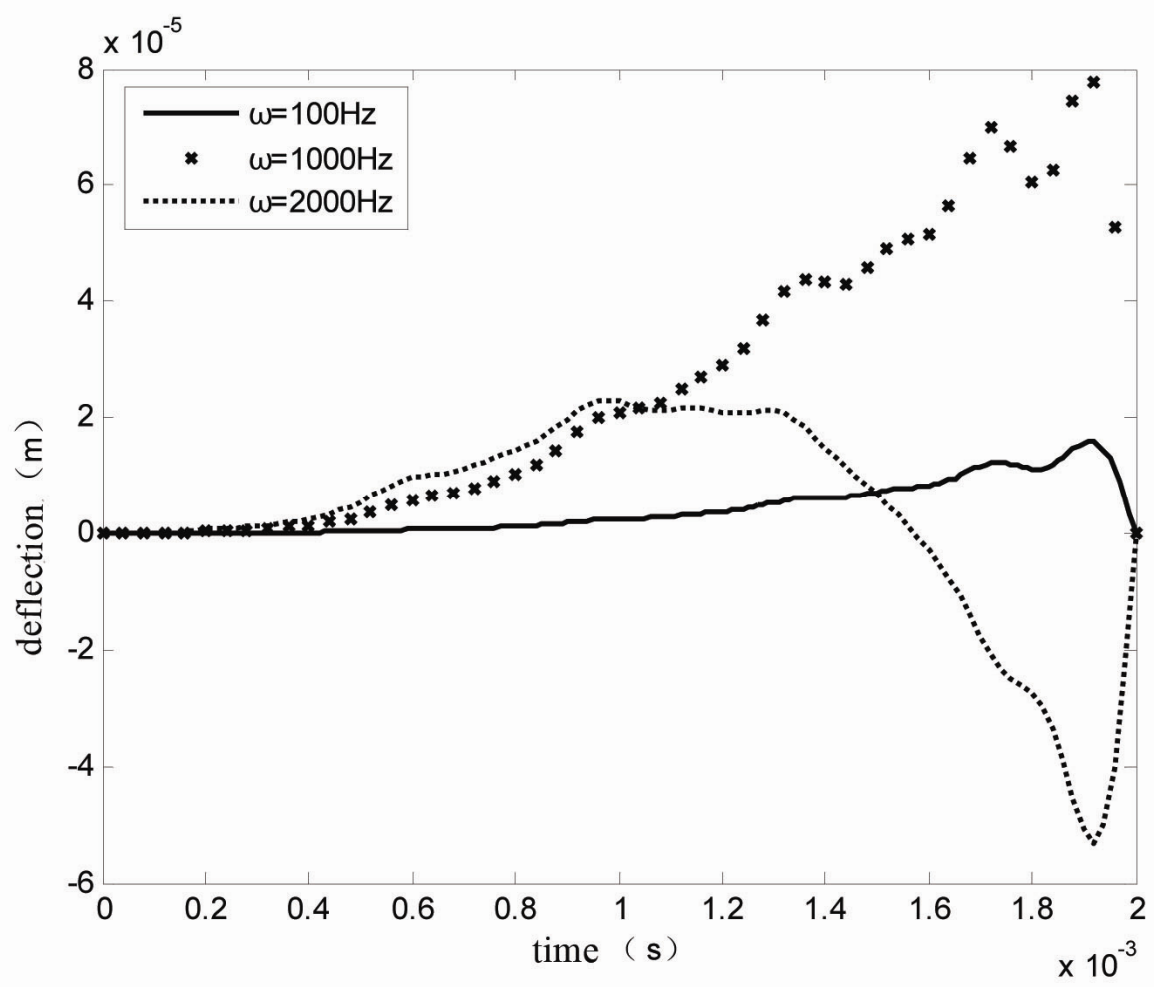

Figure 4. Node deflection-time curve at different frequencies 


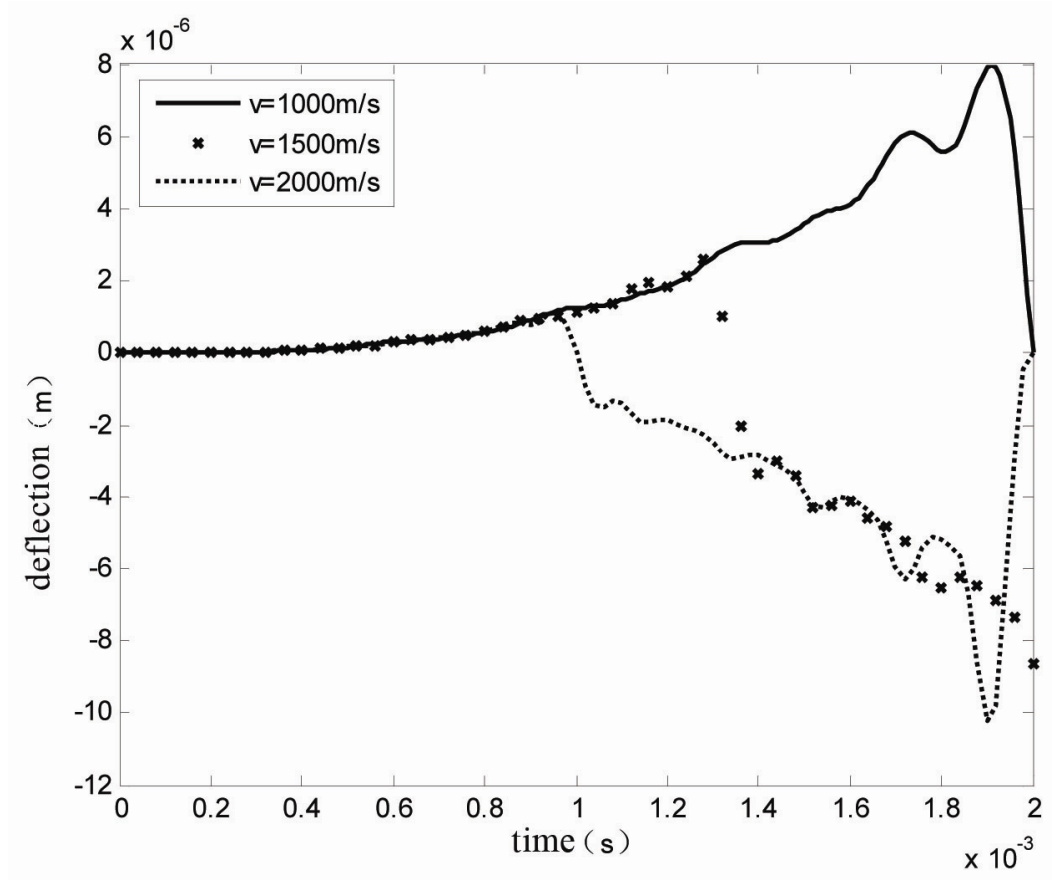

(a) Exciting frequency $\omega=50 \mathrm{~Hz}$

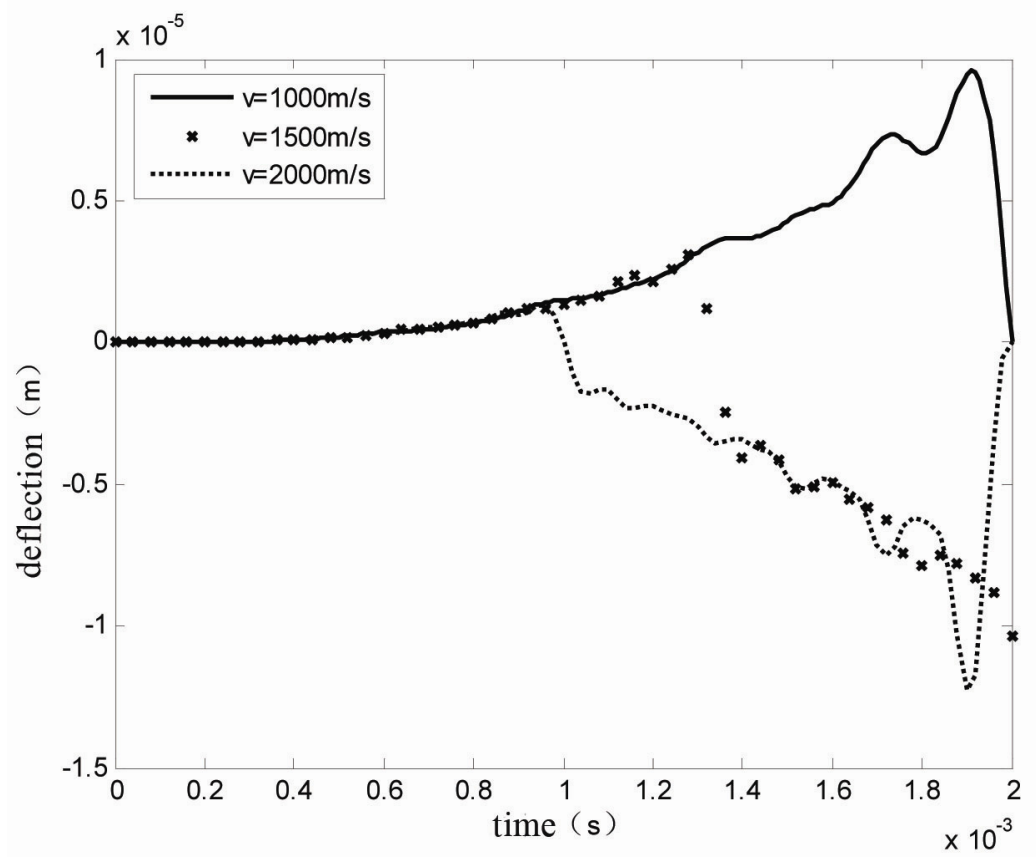

(b) Exciting frequency $\omega=60 \mathrm{~Hz}$

Figure 5. Node deflection-time curve at different speeds of load 


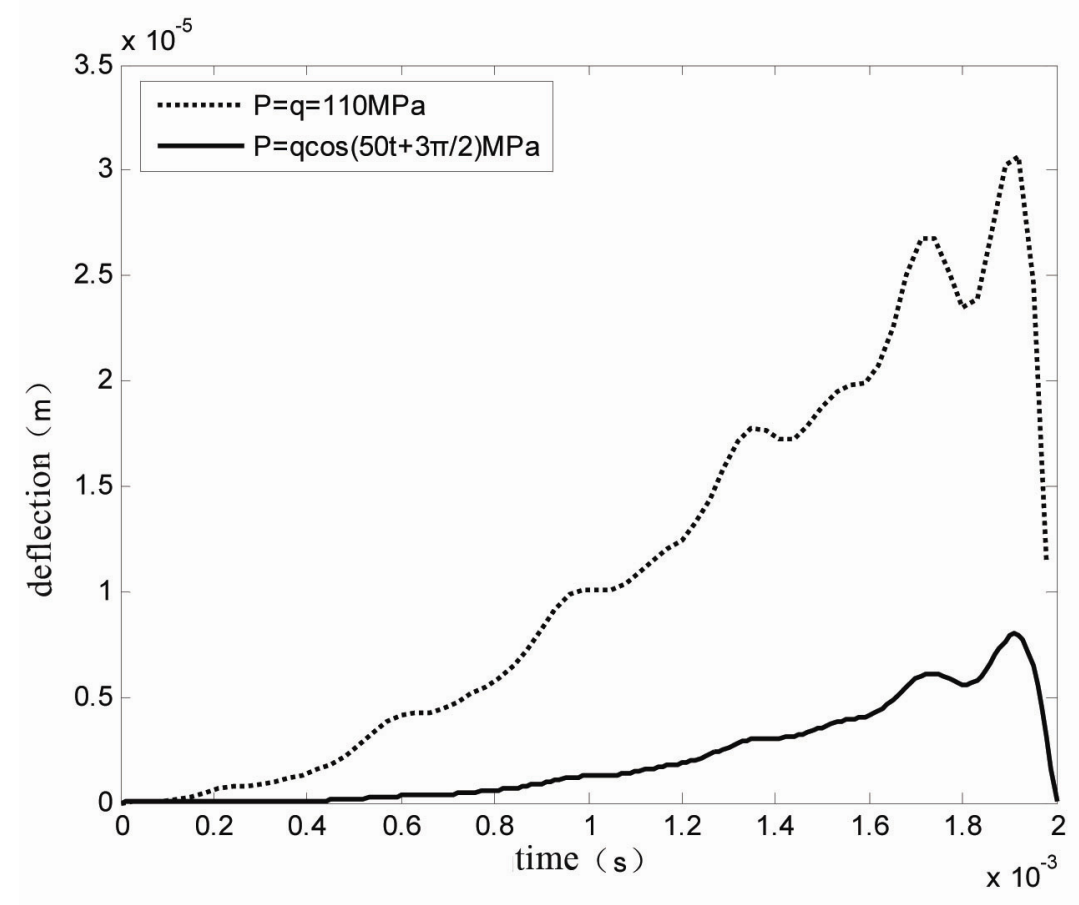

Figure 6. Node deflection-time curve at different loads

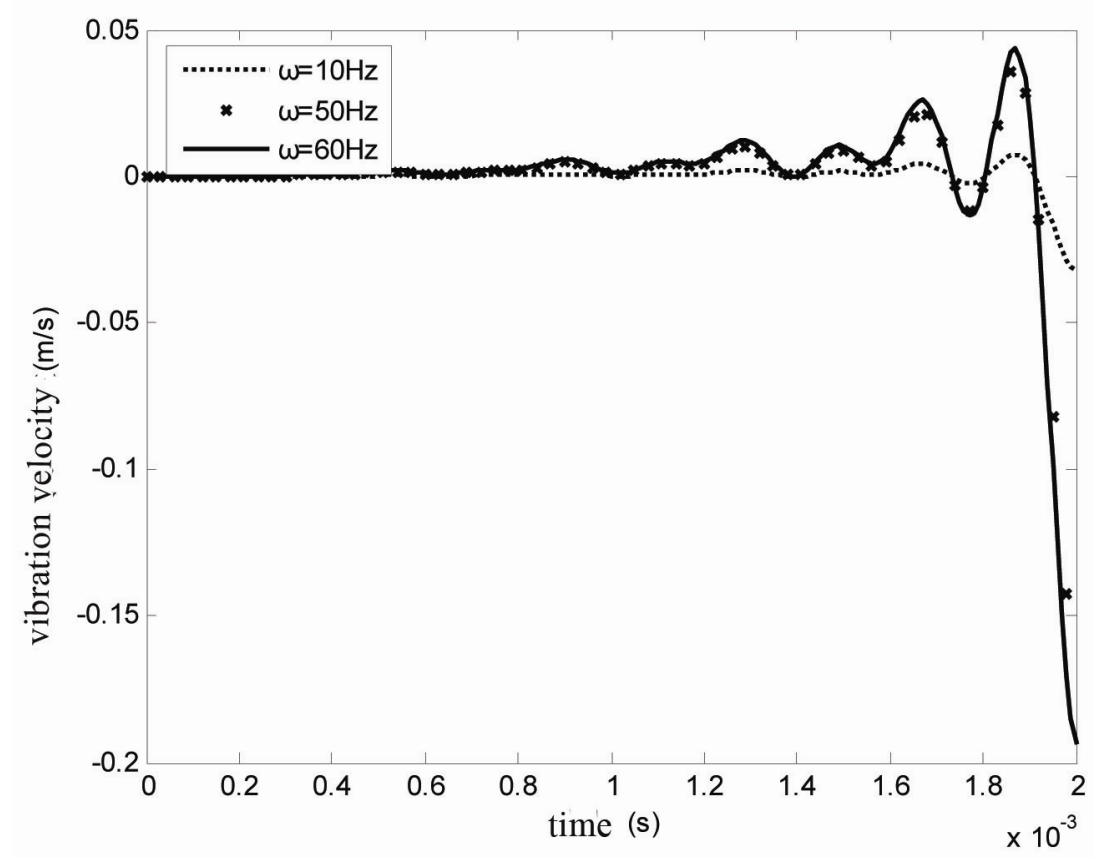

Figure 7. Vibration velocity-time curve at different frequencies 


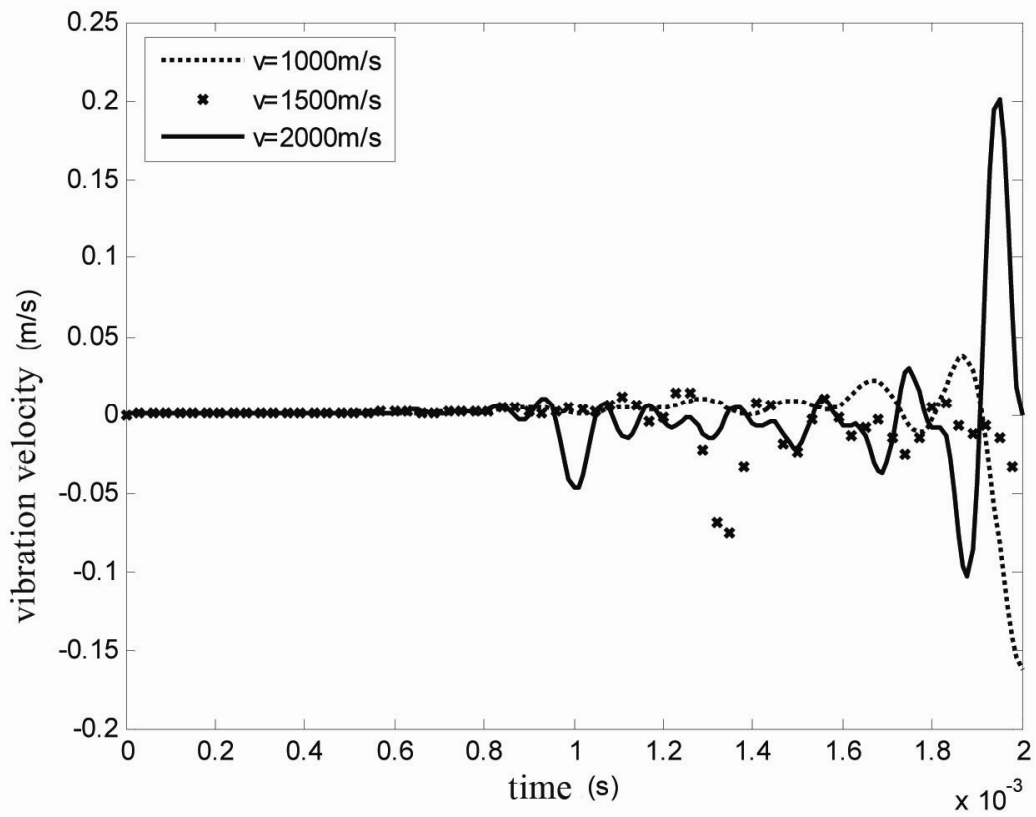

(a) Exciting frequency $\omega=50 \mathrm{~Hz}$

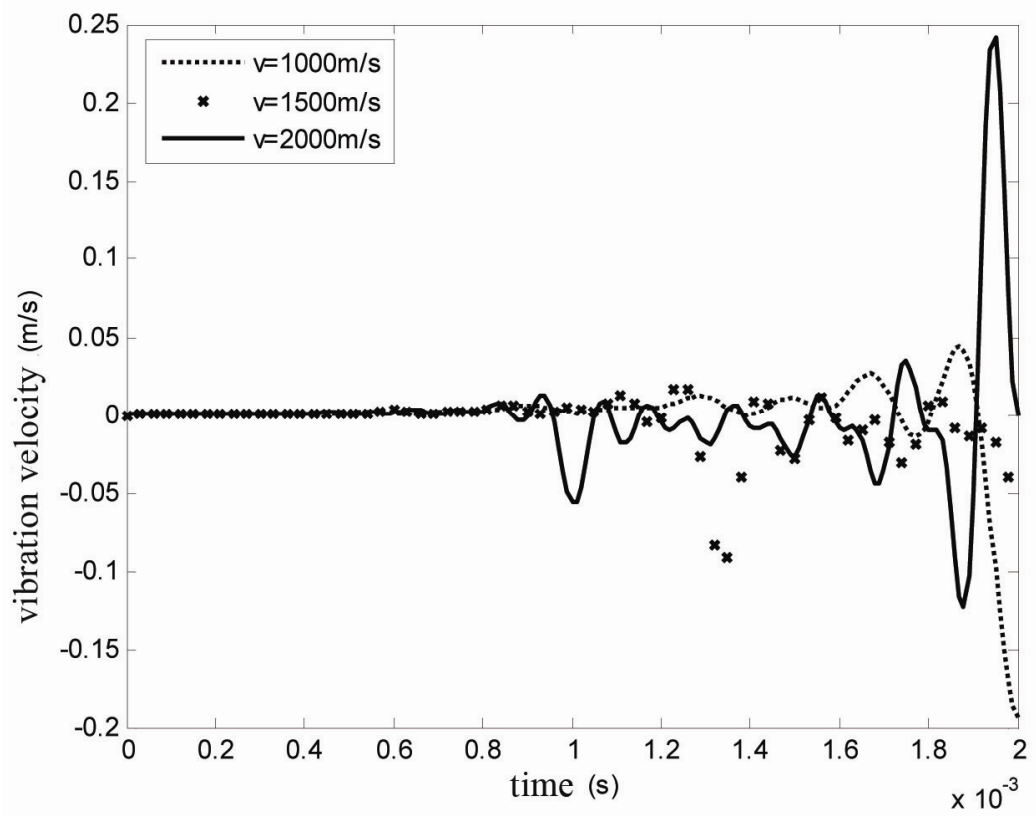

(b) Exciting frequency $\omega=60 \mathrm{~Hz}$

Figure 8. Vibration velocity-time curve at same frequencies 


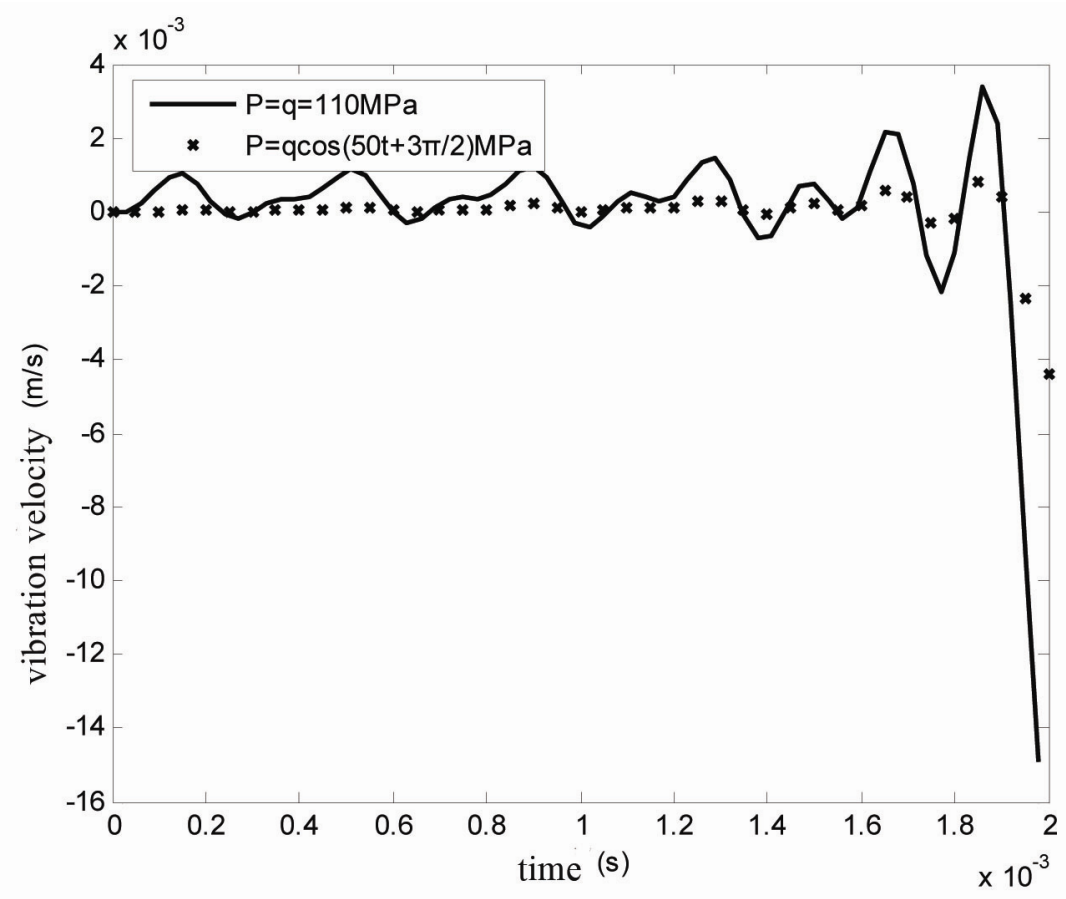

Figure 9. Vibration velocity-time curve at different loads 